Kragujevac Journal of Mathematics

Volume 45(3) (2021), Pages 393-407.

\title{
NEW GENERALIZED APOSTOL-FROBENIUS-EULER POLYNOMIALS AND THEIR MATRIX APPROACH
}

\author{
MARÍA JOSÉ ORTEGA ${ }^{1}$, WILLIAM RAMÍREZ ${ }^{1}$, AND ALEJANDRO URIELES ${ }^{2}$
}

\begin{abstract}
In this paper, we introduce a new extension of the generalized ApostolFrobenius-Euler polynomials $\mathcal{H}_{n}^{[m-1, \alpha]}(x ; c, a ; \lambda ; u)$. We give some algebraic and differential properties, as well as, relationships between this polynomials class with other polynomials and numbers. We also, introduce the generalized ApostolFrobenius-Euler polynomials matrix $U^{[m-1, \alpha]}(x ; c, a ; \lambda ; u)$ and the new generalized Apostol-Frobenius-Euler matrix $U^{[m-1, \alpha]}(c, a ; \lambda ; u)$, we deduce a product formula for $\mathcal{U}^{[m-1, \alpha]}(x ; c, a ; \lambda ; u)$ and provide some factorizations of the Apostol-Frobenius-Euler polynomial matrix $U^{[m-1, \alpha]}(x ; c, a ; \lambda ; u)$, which involving the generalized Pascal matrix.
\end{abstract}

\section{INTRODUCTION}

It is well-known that generalized Frobenius-Euler polynomial $H_{n}^{(\alpha)}(x ; u)$ of order $\alpha$ is defined by means of the following generating function

$$
\left(\frac{1-u}{e^{z}-u}\right)^{\alpha} e^{x z}=\sum_{n=0}^{\infty} H_{n}^{(\alpha)}(x ; u) \frac{z^{n}}{n !},
$$

where $u \in \mathbb{C}$ and $\alpha \in \mathbb{Z}$. Observe that $H_{n}^{(1)}(x ; u)=H_{n}(x ; u)$ denotes the classical Frobenius-Euler polynomials and $H_{n}^{(\alpha)}(0 ; u)=H_{n}^{(\alpha)}(u)$ denotes the Frobenius-Euler numbers of order $\alpha . H_{n}(x ;-1)=E_{n}(x)$ denotes the Euler polynomials (see $[2,7]$ ).

For parameters $\lambda, u \in \mathbb{C}$ and $a, b, c \in \mathbb{R}^{+}$, the Apostol type Frobenius-Euler polynomials $H_{n}(x ; \lambda ; u)$ and the generalized Apostol-type Frobenius-Euler polynomials are

Key words and phrases. Generalized Apostol-type polynomials, Apostol-Frobennius-Euler polynomials, Apostol-Bernoulli polynomials of higher order, Apostol-Genocchi polynomials of higher order, Stirling numbers of second kind, generalized Pascal matrix.

2010 Mathematics Subject Classification. Primary: 33E12. Secondary: 30H50.

DOI 10.46793/KgJMat2103.393O

Received: June 06, 2018.

Accepted: January 25, 2019. 
defined by means of the following generating functions (see [8]):

$$
\begin{aligned}
\left(\frac{1-u}{\lambda e^{z}-u}\right) e^{x z} & =\sum_{n=0}^{\infty} H_{n}(x ; \lambda ; u) \frac{z^{n}}{n !}, \\
\left(\frac{a^{z}-u}{\lambda b^{z}-u}\right)^{\alpha} c^{x z} & =\sum_{n=0}^{\infty} H_{n}^{(\alpha)}(x ; a, b, c ; \lambda ; u) \frac{z^{n}}{n !} .
\end{aligned}
$$

If we set $x=0$ and $\alpha=1$ in (1.3), we get

$$
\frac{a^{z}-u}{\lambda b^{z}-u}=\sum_{n=0}^{\infty} H_{n}(a, b, c ; \lambda ; u) \frac{z^{n}}{n !}
$$

$H_{n}(a, b, c ; u ; \lambda)$ denotes the generalized Apostol-type Frobenius-Euler numbers (see $[8])$.

In the present paper, we introduce a new class of Frobenius-Euler polynomials considering the work of [8], we give relationships between this polynomials whit other polynomials and numbers, as well as the generalized Apostol-Frobenius-euler polynomials matrix.

The paper is organized as follows. Section 2 contains the definitions of Apostoltype Frobenius-Euler and generalized Apostol-Frobenius-Euler polynomials and some auxiliary results. In Section 3, we define the generalized Apostol-type Frobenius-Euler polynomials and prove some algebraic and differential properties of them, as well as their relation with the Stirling numbers of second kind. Finally, in Section 4 we introduce the generalized Apostol-type Frobenius-Euler polynomial matrix, derive a product formula for it and give some factorizations for such a matrix, which involve summation matrices and the generalized Pascal matrix of first kind in base $c$, respectively.

\section{Previous Definitions and Notations}

Throughout this paper, we use the following standard notions: $\mathbb{N}=\{1,2, \ldots\}$, $\mathbb{N}_{0}=\{0,1,2, \ldots\}, \mathbb{Z}$ denotes the set of integers, $\mathbb{R}$ denotes the set of real numbers and $\mathbb{C}$ denotes the set of complex numbers. Furthermore, $\left(\lambda_{0}\right)=1$ and

$$
(\lambda)_{k}=\lambda(\lambda+1)(\lambda+2) \cdots(\lambda+k-1),
$$

where $k \in \mathbb{N}, \lambda \in \mathbb{C}$. For the complex logarithm, we consider the principal branch. All matrices are in $M_{n+1}(\mathbb{K})$, the set of all $(n+1) \times(n+1)$ matrices over the field $\mathbb{K}$, with $\mathbb{K}=\mathbb{R}$ or $\mathbb{C}$. Also, for $i, j$ any nonnegative integers we adopt the following convention

$$
\left(\begin{array}{l}
i \\
j
\end{array}\right)=0, \quad \text { whenever } j>i \text {. }
$$

Now, let us givel some properties of the generalized Apostol-type Frobenius-Euler polynomials and generalized Apostol-type Frobenius-Euler polynomials with parameters $\lambda, a, c$, order $\alpha$ (see $[4,8,11])$. 
Proposition 2.1. For a $m \in \mathbb{N}$, let $\left\{H_{n}^{(\alpha)}(x ; u)\right\}_{n \geq 0}$ and $\left\{H_{n}(x ; \lambda ; u)\right\}_{n \geq 0}$ be the sequences of generalized Apostol-type Frobenius-Euler polynomials, generalized FrobeniusEuler polynomials respectively. Then the following statements hold.

(a) Special values: for $n \in \mathbb{N}_{0}$,

$$
H_{n}^{(0)}(x ; u)=x^{n}
$$

(b) Summation formulas:

$$
\begin{aligned}
H_{n}^{(\alpha)}(x ; u ; a, b, c ; \lambda) & =\sum_{k=0}^{n}\left(\begin{array}{l}
n \\
k
\end{array}\right) H_{k}^{(\alpha)}(x ; u ; a, b, c ; \lambda)(x \ln c)^{n-k}, \\
H_{n}^{(\alpha+\beta)}(x+y ; u ; a, b, c ; \lambda) & =\sum_{k=0}^{n}\left(\begin{array}{l}
n \\
k
\end{array}\right) H_{k}^{(\alpha)}(x ; u ; a, b, c ; \lambda) H_{n-k}^{(\beta)}(y ; u ; a, b, c ; \lambda), \\
((x+y) \ln c)^{n} & =H_{n-k}^{(\alpha)}(y ; u ; a, b, c ; \lambda) H_{k}^{(-\alpha)}(x ; u ; a, b, c ; \lambda), \\
H_{n}^{(-\alpha)}\left(x ; u^{2} ; a^{2}, b^{2}, c^{2} ; \lambda^{2}\right) & =\sum_{k=0}^{n}\left(\begin{array}{l}
n \\
k
\end{array}\right) H_{k}^{(-\alpha)}(x ; u ; a, b, c ; \lambda) H_{n-k}^{(-\alpha)}(x ;-u ; a, b, c ; \lambda) .
\end{aligned}
$$

Definition 2.1. ([5, p. 207]). For $n \in \mathbb{N}_{0}$ and $x \in \mathbb{C}$, the Stirling numbers of second kind $S(n, k)$ are defined by means of the following expansion

$$
x^{n}=\sum_{k=0}^{n}\left(\begin{array}{l}
x \\
k
\end{array}\right) k ! S(n, k) .
$$

The Jacobi polynomials of the degree $n$ y orde $(\alpha, \beta)$, with $\alpha, \beta>-1$, the $n$-th Jacobi polynomial $P_{n}^{(\alpha, \beta)}(x)$ may be defined through Rodrigues' formula

$$
P_{n}^{(\alpha, \beta)}(x)=(1-x)^{-\alpha}(1+x)^{-\beta} \frac{(-1)^{n}}{2^{n} n !} \frac{d^{n}}{d x^{n}}\left\{(1-x)^{n+\alpha}(1+x)^{n+\alpha}\right\}
$$

and the values in the end points of the interval $[-1,1]$ is given by

$$
P_{n}^{(\alpha, \beta)}(1)=\left(\begin{array}{c}
n+\alpha \\
n
\end{array}\right), \quad P_{n}^{(\alpha, \beta)}(-1)=(-1)^{n}\left(\begin{array}{c}
n+\beta \\
n
\end{array}\right) .
$$

The relationship between the $n$-th monomial $x^{n}$ and the $n$-th Jacobi polynomial $P_{n}^{(\alpha, \beta)}(x)$ may be written as

$$
x^{n}=n ! \sum_{k=0}^{n}\left(\begin{array}{l}
n+\alpha \\
n-k
\end{array}\right)(-1)^{k} \frac{(1+\alpha+\beta+2 k)}{(1+\alpha+\beta+k)_{n+1}} P_{k}^{(\alpha, \beta)}(1-2 x) .
$$

Proposition 2.2. For $\lambda \in \mathbb{C}$ and $m \in \mathbb{N}$, let $\left\{B_{n}^{[m-1]}(x)\right\}_{n \geq 0}, \quad\left\{G_{n}(x)\right\}_{n \geq 0}$ and $\left\{\mathcal{E}_{n}(x ; \lambda)\right\}_{n \geq 0}$ be the sequences of generalized Bernoulli polynomials of level $m$, Genocchi polynomials and Apostol-Euler polynomials, respectively, we have the relationships:

(a) $[12$, Equation (4)]

$$
x^{n}=\sum_{k=0}^{n}\left(\begin{array}{l}
n \\
k
\end{array}\right) \frac{k !}{(k+m) !} B_{n-k}^{[m-1]}(x)
$$


(b) $[9$, Remark 7$]$

$$
x^{n}=\frac{1}{2(n+1)}\left[\sum_{k=0}^{n+1}\left(\begin{array}{c}
n+1 \\
k
\end{array}\right) G_{k}(x)+G_{n+1}(x)\right] ;
$$

(c) $[10$, Equation $(32)]$

$$
x^{n}=\frac{1}{2}\left[\lambda \sum_{k=0}^{n}\left(\begin{array}{l}
n \\
k
\end{array}\right) \mathcal{E}_{k}(x ; \lambda)+\mathcal{E}_{n}(x ; \lambda)\right] .
$$

Definition 2.2. Let $x$ be any nonzero real number. For $c \in \mathbb{R}^{+}$, the generalized Pascal matrix of first kind in base $c P_{c}[x]$ is an $(n+1) \times(n+1)$ matrix whose entries are given by (see $[13,14])$

$$
p_{i, j, c}(x):= \begin{cases}\left(\begin{array}{l}
i \\
j
\end{array}\right)(x \ln c)^{i-j}, & i \geq j \\
0, & \text { otherwise. }\end{cases}
$$

When $c=e$, the matrix $P_{c}[x]$ coincides with the generalized Pascal matrix of first kind $P[x]$. Furthermore, if we adopt the convention $0^{0}=1$, then $P_{c}[0]=I_{n+1}$, with $I_{n+1}=\operatorname{diag}(1,1, \ldots, 1)$.

An immediate consequence of the remarks above is the following proposition.

Proposition 2.3 (Addition Theorem of the argument). For $x, y \in \mathbb{R}$ is fulfilled

$$
P_{c}[x+y]=P_{c}[x] P_{c}[y] .
$$

Proposition 2.4. For $c \in \mathbb{R}^{+}$, let $P_{c}[x]$ be the generalized Pascal matrix of first kind in base $c$ and order $n+1$. Then the following statements hold.

(a) $P_{c}[x]$ is an invertible matrix and its inverse is given by

$$
P_{c}^{-1}[x]:=\left(P_{c}[x]\right)^{-1}=P_{c}[-x] .
$$

(e) The matrix $P_{c}[x]$ can be factorized as follows

$$
P_{c}[x]=G_{n, c}[x] G_{n-1, c}[x] \cdots G_{1, c}[x],
$$

where $G_{k, c}[x]$ is the $(n+1) \times(n+1)$ summation matrix given by

$$
G_{k, c}[x]= \begin{cases}{\left[\begin{array}{cc}
I_{n-k} & 0 \\
0 & S_{k, c}[x]
\end{array}\right],} & k=1, \ldots, n-1, \\
S_{n, c}[x], & k=n,\end{cases}
$$

being $S_{k, c}[x]$ the $(k+1) \times(k+1)$ matrix whose entries $S_{k, c}(x ; i, j)$ are given by

$$
S_{k, c}(x ; i, j, c)=\left\{\begin{array}{ll}
(x \ln c)^{i-j}, & i \geq j, \\
0, & j>i,
\end{array} \quad 0 \leq i, j \leq k .\right.
$$




\section{Generalized Apostol-Frobenius-Euler Polynomials$$
\mathcal{H}_{n}^{[m-1, \alpha]}(x ; c, a ; \lambda ; u)
$$

Definition 3.1. For $m \in \mathbb{N}, \alpha, \lambda, u \in \mathbb{C}$ and $a, c \in \mathbb{R}^{+}$, the generalized Apostol-type Frobenius-Euler polynomials in the variable $x$, parameters $c, a, \lambda$, order $\alpha$ and level $m$, are defined through the following generating function

$$
\left[\frac{\sum_{h=0}^{m-1} \frac{(z \ln a)^{h}}{h !}-u^{m}}{\lambda c^{z}-u^{m}}\right]^{\alpha} c^{x z}=\sum_{n=0}^{\infty} \mathcal{H}_{n}^{[m-1, \alpha]}(x ; c ; a ; \lambda ; u) \frac{z^{n}}{n !},
$$

where $|z|<\left|\frac{\ln \left(u^{m}\right)}{\ln (c)}-\frac{\ln (\lambda)}{\ln (c)}\right|$.

For $x=0$ we obtain, the generalized Apostol-Frobennius-Euler numbers of parameters $\lambda \in \mathbb{C}, a, c \in \mathbb{R}^{+}$, order $\alpha \in \mathbb{C}$ and level $m \in \mathbb{N}$

$$
\mathcal{H}_{n}^{[m-1, \alpha]}(c, a ; \lambda ; u):=\mathcal{H}_{n}^{[m-1, \alpha]}(0 ; c, a ; \lambda ; u) .
$$

According to the Definition 3.1, with $e=\exp (1)$, we have (1.1) and (1.2)

$$
\begin{aligned}
& \mathcal{H}_{n}^{[0, \alpha]}(x ; e, 1 ; 1 ; u)=H_{n}^{(\alpha)}(x ; \lambda ; u), \\
& \mathcal{H}_{n}^{[0,1]}(x ; e, 1 ; \lambda ; u)=H_{n}^{(1)}(x ; \lambda ; u) .
\end{aligned}
$$

Example 3.1. For any $\lambda \in \mathbb{C}, m=2, c=2, a=3, \alpha=\frac{1}{2}$ and $u=2$ the first the generalized Apostol-type Frobenius-Euler polynomials in the variable $x$, parameters $c, a, \lambda$, order $\alpha$ and level $m$ are:

$$
\begin{aligned}
\mathcal{H}_{0}^{\left[1,\left(\frac{1}{2}\right)\right]}(x ; 2,3 ; \lambda ; 2)= & \sqrt{\frac{3}{\lambda-4}}, \\
\mathcal{H}_{1}^{\left[1,\left(\frac{1}{2}\right)\right]}(x ; 2,3 ; \lambda ; 2)= & \sqrt{\frac{-3}{\lambda-4}} x\left[\frac{1}{2}\left(\frac{\ln 3}{\lambda-4}+\frac{3 \lambda \ln 2}{(\lambda-4)^{2}}\right)+x \ln 4\right], \\
\mathcal{H}_{2}^{\left[1,\left(\frac{1}{2}\right)\right]}(x ; 2,3 ; \lambda ; 2)= & \frac{1}{2} x^{2}\left[\left(\frac{-3}{4} \sqrt{\frac{-3}{\lambda-4}}\left(\frac{\ln 3}{\lambda-4}+\frac{3 \lambda \ln 2}{(\lambda-4)^{2}}\right)^{2}\right.\right. \\
& \left.+\frac{1}{2} \sqrt{\frac{-3}{\lambda-4}} \frac{-2 \ln 3 \ln 2}{(\lambda-4)^{2}}-\frac{6 \lambda^{2} \ln 4}{(\lambda-4)^{3}}+\frac{3 \lambda \ln 4}{(\lambda-4)^{2}}\right) \\
& \left.+x \ln 2 \sqrt{\frac{-3}{\lambda-4}}\left(\frac{\ln 3}{\lambda-4}+\frac{3 \ln 2}{(\lambda-4)^{4}}\right)+x^{2} \ln 4 \sqrt{\frac{-3}{\lambda-4}}\right] .
\end{aligned}
$$

Example 3.2. For any $\lambda \in \mathbb{C}, m=4, c=2, a=3, \alpha=1$ and $u=2$ the first the generalized Apostol-type Frobenius-Euler polynomials in the variable $x$, parameters $c, a, \lambda$, order $\alpha$ and level $m$ are:

$$
\mathcal{H}_{0}^{[3,1]}(x ; 2,3 ; \lambda ; 2)=\frac{-15}{\lambda-16}
$$




$$
\begin{aligned}
\mathcal{H}_{1}^{[3,1]}(x ; 2,3 ; \lambda ; 2)= & x\left[\frac{\ln 3}{\lambda-16}+\frac{\lambda 15 \ln 2}{(\lambda-16)^{2}}-x \frac{15 \ln 2}{\lambda-16}\right], \\
\mathcal{H}_{2}^{[3,1]}(x ; 2,3 ; \lambda ; 2)= & \frac{1}{2} x^{2}\left[\frac{\ln 9}{\lambda-16}-\lambda \frac{2 \ln 3 \ln 2}{(\lambda-16)^{2}}+x \frac{2 \ln 3 \ln 2}{\lambda-16}-\lambda^{2} \frac{30 \ln 4}{(\lambda-16)^{3}}\right. \\
& \left.+x \frac{30 \lambda \ln 4}{(\lambda-16)^{2}}+\lambda \frac{15 \ln 4}{(\lambda-16)^{2}}-x^{2} \frac{15 \ln 4}{\lambda-16}\right] .
\end{aligned}
$$

Example 3.3. For any $\lambda \in \mathbb{C}, m=2, c=3, a=e, \alpha=\frac{1}{3}$, and $u=5$ the first the generalized Apostol-type Frobenius-Euler polynomials in the variable $x$, parameters $c, a, \lambda$, order $\alpha$ and level $m$ are:

$$
\begin{aligned}
\mathcal{H}_{0}^{\left[1,\left(\frac{1}{3}\right)\right]}(x ; 3, e ; \lambda ; 5)= & \sqrt[3]{\frac{-24}{\lambda-25}}, \\
\mathcal{H}_{1}^{\left[1,\left(\frac{1}{3}\right)\right]}(x ; 3, e ; \lambda ; 5)= & x\left[\frac{1}{3} \sqrt[3]{\left(\frac{\lambda-25}{-24}\right)^{2}}\left(\frac{\omega}{\lambda-25}+\lambda \frac{24 \ln 3}{(\lambda-25)^{2}}\right)\right. \\
& +x \ln 3 \sqrt[3]{\left.\frac{-24}{\lambda-25}\right]}, \\
\mathcal{H}_{2}^{\left[1,\left(\frac{1}{3}\right)\right]}(x ; 3, e ; \lambda ; 5)= & \frac{1}{2} x^{2}\left[\left(\frac{2}{9} \sqrt[3]{\left(\frac{\lambda-25}{-24}\right)^{5}} \frac{\omega}{\lambda-25}+\lambda \frac{24 \ln 3}{(\lambda-25)^{2}}\right)^{2}\right. \\
& +\frac{2}{3} x \sqrt[3]{\left(\frac{\lambda-25}{-24}\right)^{2}} \ln 3\left(\frac{\omega}{\lambda-25}+\lambda \frac{24 \ln 3}{(\lambda-25)^{2}}\right) \\
& +\frac{1}{3} \sqrt[3]{\left(\frac{\lambda-25}{-24}\right)^{2}}\left(-2 \ln 3 \frac{\omega}{(\lambda-25)}-\lambda^{2} \frac{-48 \ln 9}{(\lambda-25)^{3}}\right. \\
& \left.+\lambda \frac{24 \ln 9}{(\lambda-25)^{2}}+x^{2} \ln 9 \sqrt[3]{\frac{-24}{\lambda-25}}\right]
\end{aligned}
$$

where $\omega=\ln \left(\frac{3060513257434037}{1125899906842624}\right)$.

Theorem 3.1. For $m \in \mathbb{N}$, let $\left\{\mathcal{H}_{n}^{[m-1, \alpha]}(x ; c, a ; \lambda ; u)\right\}_{n \geq 0}$ be the sequence of generalized Apostol-type Frobenius-Euler polynomials, whit parameters $\lambda, u \in \mathbb{C}$ and $a, c \in \mathbb{R}^{+}$, order $\alpha \in \mathbb{C}$ and level $m$. Then the following statements hold.

(a) For every $\alpha=0$ and $n \in \mathbb{N}_{0}$

$$
\mathcal{H}_{n}^{[m-1,0]}(x ; c ; a ; \lambda ; u)=(x \ln c)^{n} .
$$

(b) For $\alpha, \lambda \in \mathbb{C}$ and $n, k \in \mathbb{N}_{0}$, we have the relationship

$$
\mathcal{H}_{n}^{[m-1, \alpha]}(x ; c ; a ; \lambda ; u)=\sum_{k=0}^{n}\left(\begin{array}{l}
n \\
k
\end{array}\right) \mathcal{H}_{n-k}^{[m-1, \alpha]}(c ; a ; \lambda ; u)(x \ln c)^{k}
$$




$$
=\sum_{k=0}^{n}\left(\begin{array}{l}
n \\
k
\end{array}\right) \mathcal{H}_{n-k}^{[m-1, \alpha-1]}(c ; a ; \lambda ; u) \mathcal{H}_{k}^{[m-1,1]}(x ; c ; a ; \lambda ; u) .
$$

(c) Differential relations. For $m \in \mathbb{N}$ and $n, j \in \mathbb{N}_{0}$ with $0 \leq j \leq n$, we have

$$
\left[\mathcal{H}_{n}^{[m-1, \alpha]}(x ; c ; a ; \lambda ; u)\right]^{(j)}=\frac{n !}{(n-j) !}(\ln c)^{j} \mathcal{H}_{n-j}^{[m-1, \alpha]}(x ; c, a ; \lambda ; u) .
$$

(d) Integral formulas. For $m \in \mathbb{N}$, is fulfilled

$\int_{x_{0}}^{x_{1}} \mathcal{H}_{n}^{[m-1, \alpha]}(x ; c, a ; \lambda ; u) d x=\frac{\ln c}{n+1}\left[\mathcal{H}_{n+1}^{[m-1, \alpha]}\left(x_{1} ; c, a ; \lambda ; u\right)-\mathcal{H}_{n+1}^{[m-1, \alpha]}\left(x_{0} ; c, a ; \lambda ; u\right)\right]$.

(e) Addition theorem of the argument.

$$
\begin{gathered}
\mathcal{H}_{n}^{[m-1, \alpha+\beta]}(x+y ; c, a ; \lambda ; u)=\sum_{k=0}^{n}\left(\begin{array}{l}
n \\
k
\end{array}\right) \mathcal{H}_{k}^{[m-1, \alpha]}(x ; c, a ; \lambda ; u) \mathcal{H}_{n-k}^{[m-1, \beta]}(y ; c, a ; \lambda ; u), \\
\mathcal{H}_{n}^{[m-1, \alpha]}(x+y ; c, a ; \lambda ; u)=\sum_{k=0}^{n}\left(\begin{array}{l}
n \\
k
\end{array}\right) \mathcal{H}_{n-k}^{[m-1, \alpha]}(y ; c, a ; \lambda ; u)(x \ln c)^{k}, \\
((x+y) \log c)^{n}=\sum_{k=0}^{n}\left(\begin{array}{l}
n \\
k
\end{array}\right) \mathcal{H}_{n-k}^{[m-1, \alpha]}(y ; c ; a ; \lambda ; u) \mathcal{H}_{k}^{[m-1,-\alpha]}(x ; c ; a ; \lambda ; u) .
\end{gathered}
$$

Proof. (3.2) From Definition 3.1, we have

$$
\begin{aligned}
& \sum_{n=0}^{\infty} \mathcal{H}_{n}^{[m-1, \alpha+\beta]}(x+y, c, a ; \lambda ; u) \frac{t^{n}}{n !} \\
& =\left[\frac{\sum_{h=0}^{m-1} \frac{(z \ln a)^{h}}{h !}-u^{m}}{\lambda c^{z}-u^{m}}\right]^{(\alpha+\beta)} c^{(x+y) z} \\
& =\left[\frac{\sum_{h=0}^{m-1} \frac{(z \ln a)^{h}}{h !}-u^{m}}{\lambda c^{z}-u^{m}}\right]^{\alpha} c^{x z}\left[\frac{\sum_{h=0}^{m-1} \frac{(z \ln a)^{h}}{h !}-u^{m}}{\lambda c^{z}-u^{m}}\right]^{\beta} c^{y z} \\
& =\sum_{n=0}^{\infty} \mathcal{H}_{n}^{[m-1, \alpha]}(x ; c ; a ; \lambda ; u) \frac{z^{n}}{n !} \sum_{n=0}^{\infty} \mathcal{H}_{n}^{[m-1, \beta]}(y ; c ; a ; \lambda ; u) \frac{z^{n}}{n !} \\
& =\sum_{n=0}^{\infty} \sum_{k=0}^{n}\left(\begin{array}{l}
n \\
k
\end{array}\right) \mathcal{H}_{k}^{[m-1, \alpha]}(x, c, a ; \lambda ; u) \mathcal{H}_{n-k}^{[m-1, \beta]}(y, c, a ; \lambda ; u) \frac{z^{n}}{n !} .
\end{aligned}
$$

Proof. (3.4) Making an adequate modification $\beta=-\alpha$ and aplply (3.2)

$$
\sum_{n=0}^{\infty} \mathcal{H}_{n}^{[m-1, \alpha+\beta]}(x+y ; c ; a ; \lambda ; u) \frac{z^{n}}{n !}
$$




$$
\begin{aligned}
& =\left[\frac{\sum_{h=0}^{m-1} \frac{(z \ln a)^{h}}{h !}-u^{m}}{\lambda c^{z}-u^{m}}\right]^{(\alpha+\beta)} c^{(x+y) z} \\
& =\left[\frac{\sum_{h=0}^{m-1} \frac{(z \ln a)^{h}}{h !}-u^{m}}{\lambda c^{z}-u^{m}}\right]^{\alpha} c^{x z}\left[\frac{\sum_{h=0}^{m-1} \frac{(z \ln a)^{h}}{h !}-u^{m}}{\lambda c^{z}-u^{m}}\right]^{\beta} c^{y z} \\
& =\sum_{n=0}^{\infty} \mathcal{H}_{n}^{[m-1, \alpha]}(x ; c ; a ; \lambda ; u) \frac{z^{n}}{n !} \sum_{n=0}^{\infty} \mathcal{H}_{n}^{[m-1,-\alpha]}(y ; c ; a ; \lambda ; u) \frac{z^{n}}{n !} \\
& =c^{(x+y) z} \\
& =\sum_{n=0}^{\infty}((x+y) \log c)^{n} \frac{z^{n}}{n !} .
\end{aligned}
$$

Therefore, (3.4) holds.

From (2.1) and Proposition 2.2 we deduce some algebraic relations connecting the polynomials $\mathcal{H}_{n}^{[m-1, \alpha]}(x ; c, a ; \lambda ; u)$ with other families of polynomials.

Theorem 3.2. For $m \in \mathbb{N}$, the generalized Apostol-type Frobenius-Euler polynomials of level $m \mathcal{H}_{n}^{[m-1, \alpha]}(x ; c, a ; \lambda ; u)$, are related with the Jacobi polynomials $P_{n}^{(\alpha, \beta)}(x)$, by means of the identity.

$$
\begin{aligned}
& \mathcal{H}_{n}^{[m-1, \alpha]}(x+y ; c, a ; \lambda ; u) \\
= & \left.\sum_{k=0}^{n}(-1)^{k} \sum_{j=k}^{n} j !(\ln c)^{j}\left(\begin{array}{c}
j+\alpha \\
j-k
\end{array}\right)\left(\begin{array}{c}
n \\
j
\end{array}\right) \frac{(1+\alpha+\beta+2 k)}{(1+\alpha+\beta+k)_{j+1}} \mathcal{H}_{n-j}^{[m-1, \alpha]}(y ; c, a ; \lambda ; \mu ; \nu)\right) P_{k}^{(\alpha, \beta)}(1-2 x) .
\end{aligned}
$$

Proof. By substituting (2.1) into the right-hand side of (3.3) and using appropriate binomial coefficient identities (see, for instance $[1,5,6]$ ), we see that

$$
\begin{aligned}
& \mathcal{H}_{n}^{[m-1, \alpha]}(x+y ; c, a ; \lambda ; u) \\
= & \sum_{j=0}^{n}\left(\begin{array}{c}
n \\
j
\end{array}\right) \mathcal{H}_{j}^{[m-1, \alpha]}(y ; c, a ; \lambda ; u)(n-j) !(\ln c)^{n-j} \sum_{k=0}^{n-j}(-1)^{k}\left(\begin{array}{l}
n-j+\alpha \\
n-j-k
\end{array}\right) \\
& \times \frac{(1+\alpha+\beta+2 k)}{(1+\alpha+\beta+k)_{n-j+1}} P_{k}^{(\alpha, \beta)}(1-2 x) \\
= & \sum_{j=0}^{n} \sum_{k=0}^{n-j}\left(\begin{array}{c}
n \\
j
\end{array}\right) \mathcal{H}_{j}^{[m-1, \alpha]}(y ; c, a ; \lambda ; u)(n-j) !(\ln c)^{n-j}(-1)^{k}\left(\begin{array}{l}
n-j+\alpha \\
n-j-k
\end{array}\right)
\end{aligned}
$$




$$
\begin{aligned}
& \times \frac{(1+\alpha+\beta+2 k)}{(1+\alpha+\beta+k)_{n-j+1}} P_{k}^{(\alpha, \beta)}(1-2 x) \\
= & \sum_{k=0}^{n}(-1)^{k} \sum_{j=0}^{n-k}\left(\begin{array}{c}
n \\
j
\end{array}\right)\left(\begin{array}{c}
n-j+\alpha \\
n-j-k
\end{array}\right) \mathcal{H}_{j}^{[m-1, \mu]}(y ; c, a ; \lambda ; u)(n-j) !(\ln c)^{n-j} \\
& \times \frac{(1+\alpha+\beta+2 k)}{(1+\alpha+\beta+k)_{n-j+1}} P_{k}^{(\alpha, \beta)}(1-2 x) \\
= & \sum_{k=0}^{n}(-1)^{k} \sum_{j=k}^{n} j !(\ln c)^{j}\left(\begin{array}{l}
j+\alpha \\
j-k
\end{array}\right)\left(\begin{array}{c}
n \\
j
\end{array}\right) \frac{(1+\alpha+\beta+2 k)}{(1+\alpha+\beta+k)_{j+1}} \\
& \times \mathcal{H}_{n-j}^{[m-1, \alpha]}(y ; c, a ; \lambda ; u) P_{k}^{(\alpha, \beta)}(1-2 x) .
\end{aligned}
$$

Therefore, (3.5) holds.

Theorem 3.3. For $m \in \mathbb{N}$, the generalized Apostol-type Frobenius-Euler polynomials of level $m \mathcal{H}_{n}^{[m-1, \alpha]}(x ; c, a ; \lambda ; u)$, are related with the generalized Bernoulli polynomials of level $m B_{n}^{[m-1]}(x)$, by means of the following identity $\mathcal{H}_{n}^{[m-1, \alpha]}(x+y ; c, a ; \lambda ; u)=\sum_{k=0}^{n} \sum_{j=k}^{n} \frac{k !(\ln c)^{j}}{(k+m) !}\left(\begin{array}{l}n \\ j\end{array}\right)\left(\begin{array}{l}j \\ k\end{array}\right) \mathcal{H}_{n-j}^{[m-1, \alpha]}(y ; c, a ; \lambda ; \mu ; \nu) B_{j-k}^{[m-1]}(x)$.

Proof. By substituting (2.2) into the right-hand side of (3.3), it suffices to follow the proof given in Theorem 3.2, making the corresponding modifications.

Theorem 3.4. For $m \in \mathbb{N}$, the generalized Apostol-type Frobenius-Euler polynomials of level $m \mathcal{H}_{n}^{[m-1, \alpha]}(x ; c, a ; \lambda ; u)$, are related with the Genocchi polynomials $G_{n}(x)$, by means of

$$
\mathcal{H}_{n}^{[m-1, \alpha]}(x ; c, a ; \lambda ; u)
$$

$$
=\frac{1}{2} \sum_{k=0}^{n} \frac{(\ln c)^{k}}{k+1}\left[\left(\begin{array}{l}
n \\
k
\end{array}\right) \mathcal{H}_{n-k}^{[m-1, \alpha]}(y ; c, a ; \lambda ; u)+\sum_{j=k}^{n}\left(\begin{array}{l}
n \\
j
\end{array}\right)\left(\begin{array}{l}
j \\
k
\end{array}\right) \mathcal{H}_{n-j}^{[m-1, \alpha]}(y ; c, a ; \lambda ; u)(\ln c)^{j-k}\right] G_{k+1}(x) .
$$

Proof. By substituting (2.3) into the right-hand side of (3.3), we see that

$$
\begin{aligned}
& \mathcal{H}_{n}^{[m-1, \alpha]}(x ; c, a ; \lambda ; u) \\
= & \sum_{j=0}^{n}\left(\begin{array}{c}
n \\
j
\end{array}\right) \mathcal{H}_{j}^{[m-1, \alpha]}(y ; c, a ; \lambda ; u) \frac{(\ln c)^{n-j}}{2(n-j+1)}\left[\sum_{k=0}^{n-j}\left(\begin{array}{c}
n-j+1 \\
k+1
\end{array}\right) G_{k+1}(x)+G_{n-j+1}(x)\right] \\
= & \sum_{j=0}^{n}\left(\begin{array}{c}
n \\
j
\end{array}\right) \mathcal{H}_{j}^{[m-1, \alpha]}(y ; c, a ; \lambda ; u) \frac{(\ln c)^{n-j}}{2(n-j+1)} \sum_{k=0}^{n-j}\left(\begin{array}{c}
n-j+1 \\
k+1
\end{array}\right) G_{k+1}(x) \\
& +\sum_{j=0}^{n}\left(\begin{array}{c}
n \\
j
\end{array}\right) \mathcal{H}_{j}^{[m-1, \alpha]}(y ; c, a ; \lambda ; u) \frac{(\ln c)^{n-j}}{2(n-j+1)} G_{n-j+1}(x) .
\end{aligned}
$$

Then, using appropriate combinational identities and summations (see, for instance $[1,5,6])$, we obtain

$$
\mathcal{H}_{n}^{[m-1, \alpha]}(x+y ; c, a ; \lambda ; u)
$$


$=\frac{1}{2} \sum_{k=0}^{n} \frac{(\ln c)^{k}}{k+1}\left[\sum_{j=k}^{n}\left(\begin{array}{c}n \\ j\end{array}\right)\left(\begin{array}{l}j \\ k\end{array}\right) \mathcal{H}_{n-j}^{[m-1, \alpha]}(y ; c, a ; \lambda ; u)(\ln c)^{j-k}+\left(\begin{array}{l}n \\ k\end{array}\right) \mathcal{H}_{n-k}^{[m-1, \alpha]}(y ; c, a ; \lambda ; u)\right] G_{k+1}(x)$. Therefore, (3.6) holds.

Theorem 3.5. For $m \in \mathbb{N}$, the generalized Apostol-type Frobeniu-Euler polynomials of level $m \mathcal{H}_{n}^{[m-1, \alpha]}(x ; c, a ; \lambda ; u)$, are related with the Apostol-Euler polynomials $\mathcal{E}_{n}(x ; \lambda)$, by means of the following identity

(3.7) $\mathcal{H}_{n}^{[m-1, \alpha]}(x+y ; c, a ; \lambda ; u)$

$$
=\frac{1}{2} \sum_{j=0}^{n}\left(\begin{array}{c}
n \\
j
\end{array}\right)\left[\lambda \mathcal{H}_{n}^{[m-1, \alpha]}(y+1 ; c, a ; \lambda ; u)+(\ln c)^{j} \mathcal{H}_{n}^{[m-1, \alpha]}(y ; c, a ; \lambda ; u)\right] \mathcal{E}_{n-j}(x ; \lambda) .
$$

Proof. By substituting (2.4) into the right-hand side of (3.3), we can see that

$$
\begin{aligned}
& \mathcal{H}_{n}^{[m-1, \alpha]}(x+y ; c, a ; \lambda ; u) \\
= & \sum_{k=0}^{n}\left(\begin{array}{l}
n \\
k
\end{array}\right) \mathcal{H}_{k}^{[m-1, \alpha]}(y ; c, a ; \lambda ; u)(\ln c)^{n-k}\left(\frac{1}{2}\right)\left[\lambda \sum_{j=0}^{n-k}\left(\begin{array}{c}
n-k \\
j
\end{array}\right) \mathcal{E}_{j}(x ; \lambda)+\mathcal{E}_{n-k}(x ; \lambda)\right] \\
= & \sum_{k=0}^{n}\left(\begin{array}{l}
n \\
k
\end{array}\right) \mathcal{H}_{k}^{[m-1, \alpha]}(y ; c, a ; \lambda ; u)(\ln c)^{n-k}\left(\frac{\lambda}{2}\right) \sum_{j=0}^{n-k}\left(\begin{array}{c}
n-k \\
j
\end{array}\right) \mathcal{E}_{j}(x ; \lambda) \\
& +\sum_{k=0}^{n}\left(\begin{array}{l}
n \\
k
\end{array}\right) \mathcal{H}_{k}^{[m-1, \alpha]}(y ; c, a ; \lambda ; u)(\ln c)^{n-k}\left(\frac{1}{2}\right) \mathcal{E}_{n-k}(x ; \lambda) .
\end{aligned}
$$

The first sum in (3.8) becomes

$$
\begin{aligned}
& \sum_{k=0}^{n}\left(\begin{array}{c}
n \\
k
\end{array}\right) \mathcal{H}_{k}^{[m-1, \alpha]}(y ; c, a ; \lambda ; u)(\ln c)^{n-k}\left(\frac{\lambda}{2}\right) \sum_{j=0}^{n-k}\left(\begin{array}{c}
n-k \\
j
\end{array}\right) \mathcal{E}_{j}(x ; \lambda) \\
= & \sum_{k=0}^{n} \sum_{j=0}^{n-k}\left(\begin{array}{c}
n \\
k
\end{array}\right)(\ln c)^{n-k}\left(\frac{\lambda}{2}\right)\left(\begin{array}{c}
n-k \\
j
\end{array}\right) \mathcal{H}_{k}^{[m-1, \alpha]}(y ; c, a ; \lambda ; u) \mathcal{E}_{j}(x ; \lambda) \\
= & \sum_{j=0}^{n}\left(\frac{\lambda}{2}\right)\left(\begin{array}{c}
n \\
j
\end{array}\right) \mathcal{E}_{j}(x ; \lambda) \sum_{k=0}^{n-j}\left(\begin{array}{c}
n-j \\
k
\end{array}\right) \mathcal{H}_{k}^{[m-1, \alpha]}(y ; c, a ; \lambda ; u)(\ln c)^{n-k} \\
= & \sum_{j=0}^{n}\left(\frac{\lambda}{2}\right)\left(\begin{array}{c}
n \\
j
\end{array}\right) \mathcal{E}_{j}(x ; \lambda) \mathcal{H}_{n-j}^{[m-1, \alpha]}(y+1 ; c, a ; \lambda ; u) .
\end{aligned}
$$

For the second sum in (3.8), we obtain

$$
\begin{aligned}
& \sum_{k=0}^{n}\left(\begin{array}{l}
n \\
k
\end{array}\right) \mathcal{H}_{k}^{[m-1, \alpha]}(y ; c, a ; \lambda ; u)(\ln c)^{n-k}\left(\frac{1}{2}\right) \mathcal{E}_{n-k}(x ; \lambda) \\
= & \frac{1}{2} \sum_{k=0}^{n}\left(\begin{array}{l}
n \\
k
\end{array}\right) \mathcal{H}_{n-k}^{[m-1, \alpha]}(y ; c, a ; \lambda ; u)(\ln c)^{k} \mathcal{E}_{k}(x ; \lambda) .
\end{aligned}
$$


Combining (3.9) and (3.10) we get

$$
\begin{aligned}
& \mathcal{H}_{n}^{[m-1, \alpha]}(x+y ; c, a ; \lambda ; u) \\
= & \left(\frac{\lambda}{2}\right) \sum_{j=0}^{n}\left(\begin{array}{c}
n \\
j
\end{array}\right) \mathcal{E}_{j}(x ; \lambda) \mathcal{H}_{n-j}^{[m-1, \alpha]}(y+1 ; c, a ; \lambda ; u) \\
& +\frac{1}{2} \sum_{j=0}^{n}\left(\begin{array}{c}
n \\
j
\end{array}\right) \mathcal{H}_{n-j}^{[m-1, \alpha]}(y ; c, a ; \lambda ; u)(\ln c)^{j} \mathcal{E}_{j}(x ; \lambda) \\
= & \frac{1}{2} \sum_{j=0}^{n}\left(\begin{array}{c}
n \\
j
\end{array}\right)\left[\lambda \mathcal{H}_{n}^{[m-1, \alpha]}(y+1 ; c, a ; \lambda ; u)+(\ln c)^{j} \mathcal{H}_{n}^{[m-1, \alpha]}(y ; c, a ; \lambda ; u)\right] \mathcal{E}_{n-j}(x ; \lambda) .
\end{aligned}
$$

Therefore, (3.7) holds.

Proposition 3.1. For $m \in \mathbb{N}, \alpha, \lambda, u, \in \mathbb{C}, a, c \in \mathbb{R}^{+}$and $n \in \mathbb{N}_{0}$, we have

$$
\begin{aligned}
\mathcal{H}_{n}^{[m-1, \alpha]}(x+y ; c, a ; \lambda ; u) & =\sum_{k=0}^{n} k !\left(\begin{array}{l}
x \\
k
\end{array}\right) \sum_{j=0}^{n-k}\left(\begin{array}{c}
n \\
j
\end{array}\right) \mathcal{H}_{j}^{[m-1, \alpha]}(y ; c, a ; \lambda ; u)(\ln c)^{n-j} S(n-j, k) \\
& =\sum_{k=0}^{n} k !\left(\begin{array}{l}
x \\
k
\end{array}\right) \sum_{j=k}^{n}\left(\begin{array}{c}
n \\
n-j
\end{array}\right) \mathcal{H}_{n-j}^{[m-1, \alpha]}(y ; c, a ; \lambda ; u)(\ln c)^{j} S(j, k) .
\end{aligned}
$$

\section{The Generalized Apostol-Frobenius-Euler Polynomials Matrix}

Definition 4.1. The generalized $(n+1) \times(n+1)$ Apostol-Frobenius-Euler polynomials matrix $\mathcal{U}^{[m-1, \alpha]}(x ; c, a ; \lambda ; u)$ with $m \in \mathbb{N}, \alpha, \lambda, u \in \mathbb{C}$ and $a, c$ positive real numbers is defined by

$$
\mathcal{U}_{i, j}^{[m-1, \alpha]}(x ; c, a ; \lambda ; u)= \begin{cases}\left(\begin{array}{l}
i \\
j
\end{array}\right) \mathcal{H}_{i-j}^{[m-1, \alpha]}(x ; c, a ; \lambda ; u), & i \geq j \\
0, & \text { otherwise. }\end{cases}
$$

While, the matrices

$$
\begin{aligned}
\mathcal{U}^{[m-1]}(x ; c, a ; \lambda ; u) & :=\mathcal{U}^{[m-1,1]}(x ; c, a ; \lambda ; u), \\
\mathcal{U}^{[m-1]}(c, a ; \lambda ; u) & :=\mathcal{U}^{[m-1]}(0 ; c, a ; \lambda ; u)
\end{aligned}
$$

are called the Apostol-Frobenius-Euler polynomial matrix and the Apostol-FrobeniusEuler matrix, respectively.

Since $\mathcal{H}_{n}^{[m-1,0]}(x ; c, a ; \lambda ; u)=(x \ln (c))^{n}$, we have $\mathcal{U}^{[m-1,0]}(x ; c, a ; \lambda ; u)=P_{c}[x]$. It is clear that (3.3) yields the following matrix identity:

$$
\mathcal{U}^{[m-1, \alpha]}(x+y ; c, a ; \lambda ; u)=\mathcal{U}^{[m-1, \alpha]}(y ; c, a ; \lambda ; u) P_{c}[x] .
$$

Theorem 4.1. For a fixed $m \in \mathbb{N}$, let $\left\{\mathcal{H}_{n}^{[m-1, \alpha]}(x ; c, a ; \lambda ; u)\right\}_{n \geq 0}$ and $\left\{\mathcal{H}_{n}^{[m-1, \beta]}(x ; c, a ; \lambda ; u)\right\}_{n \geq 0}$ be the sequences of generalized Apostol-type Frobenius-Euler 
polynomials in the variable $x$, parameters $\lambda, u \in \mathbb{C}, a, c \in \mathbb{R}^{+}$, order $\alpha \in \mathbb{C}$ and level $m$. Then satisfies the following product formula:

$$
\begin{aligned}
\mathcal{U}^{[m-1, \alpha+\beta]}(x+y ; c, a ; \lambda ; u) & =U^{[m-1, \alpha]}(x ; c, a ; \lambda ; u) U^{[m-1, \beta]}(y ; c, a ; \lambda ; u) \\
& =U^{[m-1, \beta]}(x ; c, a ; \lambda ; u) U^{[m-1, \alpha]}(y ; c, a ; \lambda ; u) \\
& =U^{[m-1, \alpha]}(y ; c, a ; \lambda ; u) U^{[m-1, \beta]}(x ; c, a ; \lambda ; u) .
\end{aligned}
$$

Proof. Let $B_{i, j, c}^{[m-1, \alpha, \beta]}(a ; \lambda ; u)(x, y)$ be the $(i, j)$-th entry of the matrix product $\mathcal{U}^{[m-1, \alpha]}(x ; c, a ; \lambda ; u) \mathcal{U}^{[m-1, \beta]}(y ; c, a ; \lambda ; u)$, then by the addition formula (3.2) we have

$$
\begin{aligned}
B_{i, j, c}^{[m-1, \alpha, \beta]}(a ; \lambda ; u)(x, y) & =\sum_{k=0}^{n}\left(\begin{array}{c}
i \\
k
\end{array}\right) \mathcal{H}_{i-k}^{[m-1, \alpha]}(x ; c, a ; \lambda ; u)\left(\begin{array}{c}
k \\
j
\end{array}\right) \mathcal{H}_{k-j}^{[m-1, \beta]}(y ; c, a ; \lambda ; u) \\
& =\sum_{k=j}^{i}\left(\begin{array}{c}
i \\
k
\end{array}\right) \mathcal{H}_{i-k}^{[m-1, \alpha]}(x ; c, a ; \lambda ; u)\left(\begin{array}{c}
k \\
j
\end{array}\right) \mathcal{H}_{k-j}^{[m-1, \beta]}(y ; c, a ; \lambda ; u) \\
& =\sum_{k=j}^{i}\left(\begin{array}{c}
i \\
j
\end{array}\right)\left(\begin{array}{c}
i-j \\
i-k
\end{array}\right) \mathcal{H}_{i-k}^{[m-1, \alpha]}(x ; c, a ; \lambda ; u) \mathcal{H}_{k-j}^{[m-1, \beta]}(y ; c, a ; \lambda ; u) \\
& =\left(\begin{array}{c}
i \\
j
\end{array}\right) \sum_{k=0}^{i-j}\left(\begin{array}{c}
i-j \\
k
\end{array}\right) \mathcal{H}_{i-j-k}^{[m-1, \alpha]}(x ; c, a ; \lambda ; u) \mathcal{H}_{k}^{[m-1, \beta]}(y ; c, a ; \lambda ; u) \\
& =\left(\begin{array}{c}
i \\
j
\end{array}\right) \mathcal{H}_{i-j}^{[m-1, \alpha+\beta]}(x+y ; c, a ; \lambda ; u),
\end{aligned}
$$

which implies the first equality of the theorem. The second and third equalities of can be derived in a similar way.

Corollary 4.1. For a fixed $m \in \mathbb{N}$, let $\left\{\mathcal{H}_{n}^{[m-1, \alpha]}(x ; c, a ; \lambda ; u)\right\}_{n \geq 0}$ and $\left\{\mathcal{H}_{n}^{[m-1, \beta]}(x ; c, a ; \lambda ; u)\right\}_{n \geq 0}$ be the sequences of generalized Apostol-type Frobenius-Euler polynomials in the variable $x$, parameters $\lambda, u \in \mathbb{C}, a, c \in \mathbb{R}^{+}$, order $\alpha \in \mathbb{C}$ and level $m$ and $P_{c}[x]$ the generalized Pascal matrix of first kind in base $c$. Then

$$
\begin{aligned}
\mathcal{U}^{[m-1, \alpha]}(x+y ; c, a ; \lambda ; u) & =\mathcal{U}^{[m-1, \alpha]}(x ; c, a ; \lambda ; u) P_{c}[y] \\
& =P_{c}[x] \mathcal{U}^{[m-1, \alpha]}(y ; c, a ; \lambda ; u) \\
& =\mathcal{U}^{[m-1, \alpha]}(y ; c, a ; \lambda ; u) P_{c}[x] .
\end{aligned}
$$

In particular,

$$
\begin{aligned}
\mathcal{U}^{[m-1]}(x+y ; c, a ; \lambda ; u) & =P_{c}[x] \bigcup^{[m-1]}(y ; c, a ; \lambda ; u) \\
& =P_{c}[y] \mathcal{U}^{[m-1]}(x ; c, a ; \lambda ; u) .
\end{aligned}
$$

Proof. The substitution $\beta=0$ into (4.1) yields

$$
\mathcal{U}^{[m-1, \alpha]}(x+y ; c, a ; \lambda ; u)=\mathcal{U}^{[m-1, \alpha]}(x ; c, a ; \lambda ; u) \mathcal{U}^{[m-1,0]}(y ; c, a ; \lambda ; u) .
$$

Since $\mathcal{U}^{[m-1,0]}(y ; c, a ; \lambda ; u)=P_{c}[y]$, we obtain

$$
\mathcal{U}^{[m-1, \alpha]}(x+y ; c, a ; \lambda ; u)=\mathcal{U}^{[m-1, \alpha]}(x ; c, a ; \lambda ; u) P_{c}[y] .
$$


A similar argument allows to show that

$$
\begin{aligned}
\mathcal{U}^{[m-1, \alpha]}(x+y ; c, a ; \lambda ; u) & =P_{c}[x] \bigcup^{[m-1, \alpha]}(y ; c, a ; \lambda ; u) \\
& =\bigcup^{[m-1, \alpha]}(y ; c, a ; \lambda ; u) P_{c}[x]
\end{aligned}
$$

Finally, the substitution $\alpha=1$ into (4.2) and its combination with the previous equations completes the proof.

Using the relation (2.5) and Corollary 4.1 we obtain the following factorization for $\mathcal{U}^{[m-1, \alpha]}(x+y ; c, a ; \lambda ; u)$ in terms of summation matrices.

$$
\mathcal{U}^{[m-1, \alpha]}(x+y ; c, a ; \lambda ; u)=\mathcal{U}^{[m-1, \alpha]}(x ; c, a ; \lambda ; u) G_{n, c}[y] G_{n-1, c}[y] \cdots G_{1, c}[y] .
$$

Under the appropriate choice on the parameters, level and order, it is possible to provide some illustrative examples of the generalized Apostol-Frobenius-Euler polynomials matrices.

Example 4.1. For $m=1, c=a=e=\exp (1), \alpha=1, \lambda=-1$, The first four polynomials $\mathcal{H}_{k}^{[1-1,1]}(x ; e, e ; 1 ; u), k=0,1,2,3$ are

$$
\begin{aligned}
& \mathcal{H}_{0}^{[1-1,1]}(x ; e, e ; 1 ; u)=1, \\
& \mathcal{H}_{1}^{[1-1,1]}(x ; e, e ; 1 ; u)=x-\frac{1}{1-u}, \\
& \mathcal{H}_{2}^{[1-1,1]}(x ; e, e ; 1 ; u)=x^{2}-\frac{2}{1-u} x+\frac{1+u}{(1-u)^{2}}, \\
& \mathcal{H}_{3}^{[1-1,1]}(x ; e, e ; 1 ; u)=x^{3}-\frac{3}{1-u} x^{2}+\frac{3(1+u)}{(1-u)^{2}} x-\frac{u^{2}+4 u+1}{(1-u)^{3}} .
\end{aligned}
$$

Hence, for $n=3$, we have

$$
\mathcal{U}^{[m-1,1]}(x ; e, e ; 1 ; u)=\left[\begin{array}{cccc}
1 & 0 & 0 & 0 \\
u_{10} & 1 & 0 & 0 \\
u_{20} & u_{21} & 1 & 0 \\
u_{30} & u_{31} & u_{32} & 1
\end{array}\right]
$$

where

$$
\begin{aligned}
& u_{10}=u_{21}=u_{32}=\mathcal{H}_{1}^{[1-1,1]}(x ; e, e ; 1 ; u), \\
& u_{20}=u_{31}=\mathcal{H}_{2}^{[1-1,1]}(x ; e, e ; 1 ; u), \\
& u_{30}=\mathcal{H}_{3}^{[1-1,1]}(x ; e, e ; 1 ; u) .
\end{aligned}
$$


Example 4.2. For $m=1, c=a=e=\exp (1), \lambda=1$ and $u=-1$, The first four polynomials $\mathcal{H}_{k}^{[1-1, \alpha]}(x ; e, e ; 1 ;-1), k=0,1,2,3$, are

$$
\begin{aligned}
& \mathcal{H}_{0}^{[1-1, \alpha]}(x ; e, e ; 1 ;-1)=1, \\
& \mathcal{H}_{1}^{[1-1, \alpha]}(x ; e, e ; 1 ;-1)=x-\frac{\alpha}{2}, \\
& \mathcal{H}_{2}^{[1-1, \alpha]}(x ; e, e ; 1 ;-1)=x^{2}-\alpha x+\frac{\alpha(\alpha-1)}{4}, \\
& \mathcal{H}_{3}^{[1-1, \alpha]}(x ; e, e ; 1 ;-1)=x^{3}-\frac{3 \alpha}{2} x^{2}+\frac{3 \alpha(\alpha-1)}{4} x-\frac{3 \alpha^{2}(\alpha-1)}{8} .
\end{aligned}
$$

Then, for $n=3$, we have

$$
\mathcal{U}^{[m-1, \alpha]}(x ; e, e ; 1 ;-1)=\left[\begin{array}{cccc}
1 & 0 & 0 & 0 \\
u_{10} & 1 & 0 & 0 \\
u_{20} & 2 u_{21} & 1 & 0 \\
u_{30} & 3 u_{31} & 3 u_{32} & 1
\end{array}\right],
$$

where

$$
\begin{aligned}
& u_{10}=u_{21}=u_{32}=\mathcal{H}_{1}^{[1-1, \alpha]}(x ; e, e ; 1 ;-1), \\
& u_{20}=u_{31}=\mathcal{H}_{2}^{[1-1, \alpha]}(x ; e, e ; 1 ;-1), \\
& u_{30}=\mathcal{H}_{3}^{[1-1, \alpha]}(x ; e, e ; 1 ;-1) .
\end{aligned}
$$

Example 4.3. For $\lambda \in \mathbb{C}, m=c=2, a=3, \alpha=\frac{1}{2}, u=2$, we have the Example 3.1. Therefore,

$$
\mathcal{U}^{\left[1, \frac{1}{2}\right]}(x ; 2,3 ; \lambda ; 2)=\left[\begin{array}{ccc}
\sqrt{\frac{3}{\lambda-4}} & 0 & 0 \\
\mathcal{H}_{1}^{\left[1,\left(\frac{1}{2}\right)\right]}(x ; 2,3 ; \lambda ; 2) & \sqrt{\frac{3}{\lambda-4}} & 0 \\
\frac{32}{\sqrt{1+\lambda}} & 0 & 0 \\
\mathcal{H}_{2}^{\left[1,\left(\frac{1}{2}\right)\right]}(x ; 2,3 ; \lambda ; 2) & 2 \mathcal{H}_{1}^{\left[1,\left(\frac{1}{2}\right)\right]}(x ; 2,3 ; \lambda ; 2) & \sqrt{\frac{3}{\lambda-4}}
\end{array}\right] .
$$

\section{REFERENCES}

[1] R. Askey, Orthogonal Polynomials and Special Functions, Regional Conference Series in Applied Mathematics, SIAM. J. W. Arrowsmith Ltd., Bristol, England, 1975.

[2] L. Carlitz, Eulerian numbers and polynomials, Math. Mag. 32 (1959), 247-260.

[3] G. Call and D. J. Velleman, Pascal's matrices, Amer. Math. Monthly 100 (1993), 372-376.

[4] L. Castilla, W. Ramírez and A. Urieles, An extended generalized q-extensions for the Apostol type polynomials, Abstr. Appl. Anal. 2018 (2018), 1-13.

[5] L. Comtet, Advanced Combinatorics: The Art of Finite and Infinite Expansions, Reidel, Dordrecht, Boston, 1974.

[6] R. L. Graham, D. E. Knuth and O. Patashnik, Concrete Mathematics, Addison-Wesley, New York, 1994.

[7] L. Hernández, Y. Quintana and A. Urieles, About extensions of generalized Apostol-type polynomials, Results Math. 68 (2015), 203-225. 
[8] B. Kurt and Y. Simsek, On the generalized Apostol-type Frobenius-Euler polynomials, Adv. Difference Equ. 2013 (2013), 1-9.

[9] Q. M. Luo, Extensions of the Genocchi polynomials and its Fourier expansions and integral representations, Osaka J. Math. 48 (2011), 291-309.

[10] Q. M. Luo and H. M. Srivastava, Some relationships between the Apostol-Bernoulli and ApostolEuler polynomials, Comput. Math. Appl. 51 (2006), 631-642.

[11] P. Natalini and A. Bernardini, A generalization of the Bernoulli polynomials, J. Appl. Math. 3 (2003), 155-163.

[12] Y. Quintana, W. Ramírez and A. Urieles, On an operational matrix method based on generalized Bernoulli polynomials of level $m$, Calcolo 55 (2018), 23-40.

[13] Y. Quintana, W. Ramírez and A. Urieles, Generalized Apostol-type polynomial matrix and its algebraic properties. Math. Repor. 21(2) (2019).

[14] Z. Zhang and J. Wang, Bernoulli matrix and its algebraic properties, Discrete Appl. Math. 154 (2006), 1622-1632.

${ }^{1}$ GICNEX,

UNIVERSIDAD DE LA COSTA,

BARRANQUILLA-COLOMBIA

Email address: mortega22@cuc.edu.co

Email address: wramirez4@cuc.edu.co

${ }^{2}$ Department of MATHEMATiCs,

UNIVERSIDAD DEL ATLÁNTICO,

KM 7 víA Pto. BARRANQUilla-Colombia

Email address: alejandrourieles@mail.uniatlantico.edu.co 